\title{
Quando si moltiplicava per gelosia
}

\author{
di Stefania Funari \\ e Marco Li Calzi
}

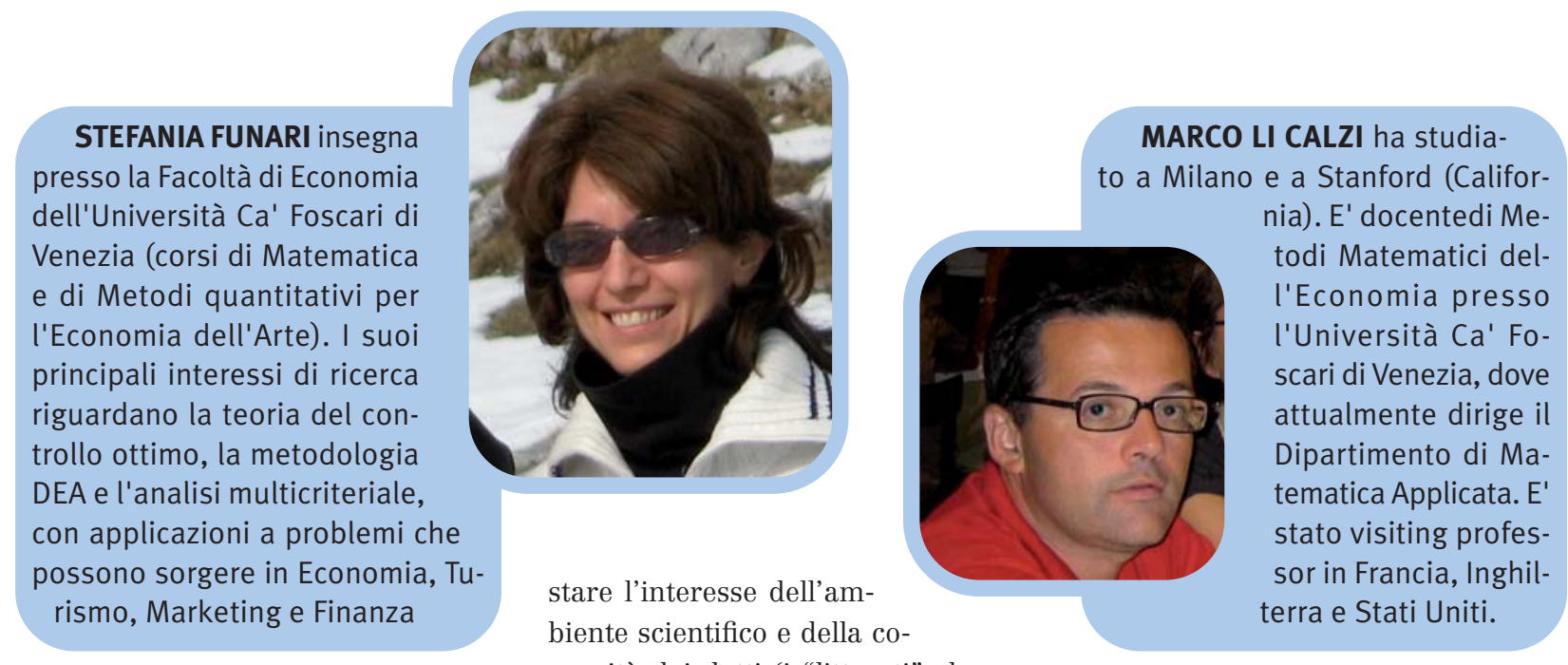

Se pensiamo ai protagonisti della MAmunità dei dotti (i "litterati" che padroneggiavano il latino). Il lavoro di Fra Luca Pacioli si proponeva di illutemATICA FRA IL XV ED ILXVI SECOLO, LA No- Strare "de ciascun atto operativo suoi STRA ATTENZIONE SI PONE SICURAMENTE Su LuCA PACIOLI e la sua famosa Summa de arithmetica, geometria, proportioni et proportionalita che venne stampata a Venezia nel 1494.

La Summa costituì un punto di riferimento per i matematici del Rinascimento, in quanto raccoglieva in un unico volume le conoscenze matematiche, che prima erano disperse in vari manoscritti, elaborate a partire dal Liber Abaci di Leonardo Pisano fino al XV secolo. L'opera non si limitò a de-

fondamenti secondo li antichi e ancor moderni philosophi”. Insieme alla scelta di far uso della "materna e vernacula lengua”, questo rese la Summa comprensibile e utile anche ai tecnici che praticavano un'arte o un mestiere (i “pratici vulgari”), fornendo uno snodo importante fra la Matematica teorica e quella pratica della bottega d'abaco. Già prima della pubblicazione della Summa, grazie all'invenzione della stampa a caratteri mobili, si erano diffusi vari manuali di Aritmetica pratica scritti in volgare. Senza vantare grande rilevanza scientifica o particolare originalità di contenuto, avevano tuttavia contribuito efficacemente alla divulgazione della Matematica e alla diffusione della cultura quantitativa. Sempre a Venezia, città tra le più attive nella produzione di libri a stampa tra il XV e il XVI secolo, nel 1484 (10 anni prima della Summa) fu pubblicata l' Arithmetica di Pietro Borghi, un'opera che ebbe notevole successo divulgativo. La prima Aritmetica stampata, Larte de labbacho di autore anonimo, era invece apparsa a Treviso nel 1478 e per questa ragione 


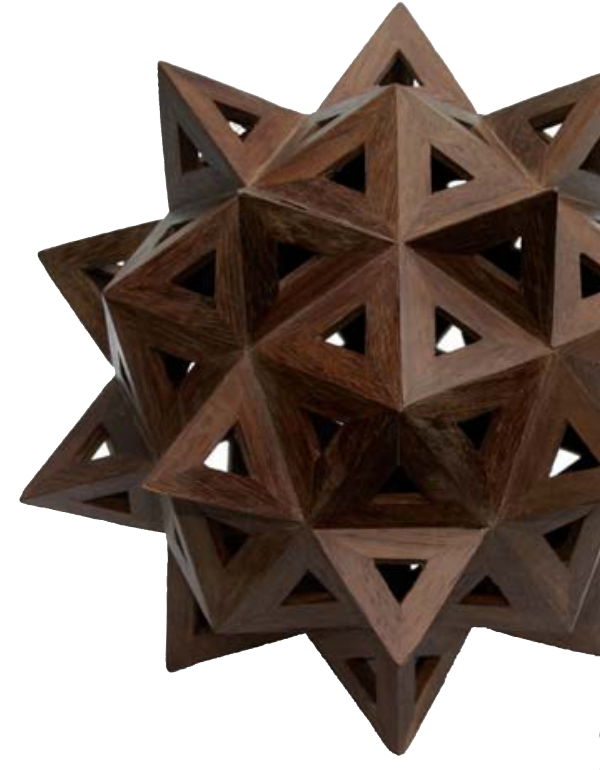

- Dida dida dida dida dida dida dida

è appunto ricordata come l' Aritmetica di Treviso. Era rivolta "a ciascheduno che vuole usare larte de la merchandantia chiamata vulgarmente larte de labbacho", a conferma dell'utilizzo pratico della Matematica nelle transazioni commerciali. Larte de labbacho e i vari manuali di Aritmetica pubblicati verso la fine del XV secolo erano fondamentalmente edizioni a stampa dei precedenti trattati d'abaco. Ne riprendevano la struttura, dedicando parti di contenuto alle definizioni dei principali concetti e operazioni, alla presentazione del sistema numerico e alla discussione di problemi reali relativi ad esempio alle operazioni di scambio, alla ripartizione fra due o più soci dei guadagni ottenuti su un certo capitale (problemi "di compagnie”), al calcolo degli interessi (“del meritare e scontare a capo d'anno”), al calcolo delle percentuali di metalli nelle leghe. In questi manuali, ampio spazio era anche riservato alla presentazione delle operazioni aritmetiche e alla verifica della loro correttezza (la famosa prova del nove!). D'altra parte, è proprio dall'esattezza dei calcoli che spesso dipendeva il buon fine di un'o- perazione commerciale. Così ad esempio veniva definita la moltiplicazione nel Larte de labbacho: "Attendi lettore al quarto atto zoe. al moltiplicare. Per intelligentia del quale el $e$ de savere. che moltiplicare uno numero [...] per uno altro: non e altro: che do numeri propositi: trovare uno terzo numero: el quale tante volte contien uno de quelli numeri: quante unitade sono nel altro [...] Intendi bene. che nella moltiplicatione sono principalmente do numeri necessarii .zoe el numero moltiplicatore et el numero de fir moltiplicato."

Esistevano diversi metodi pratici per moltiplicare e dividere ("partire") due numeri. I metodi più utilizzati per la divisione erano il partir per danda, che usiamo ancora oggi, e il partir per battello (o per galera). Si conosceva poi la moltiplicazione per bericuocolo (detta anche per organetto e chiamata dai veneziani per scachero), che corrisponde all'algoritmo attuale, la moltiplicazione per quadrilatero e le regole per crocetta, alla russa, a castelluccio e per scapezzo. I diversi nomi richiamavano la figura che, di volta in volta, scaturiva dallo schema grafico che rap-

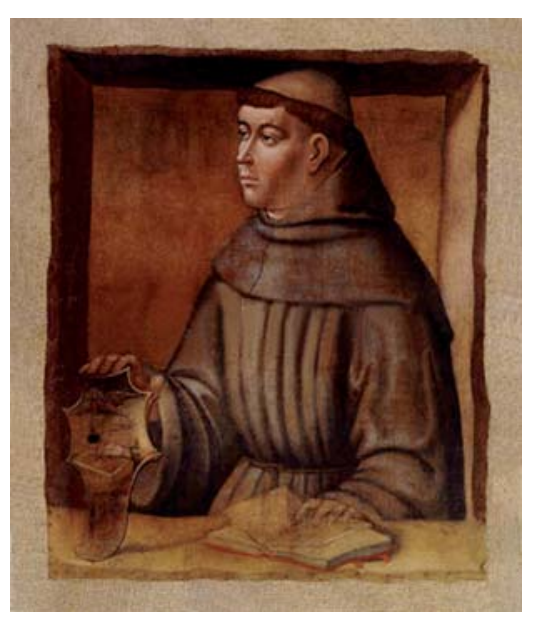

- Dida dida dida dida dida dida dida presentava la procedura risolutiva oppure identificavano la provenienza del metodo. Non c'era un metodo migliore di un altro per moltiplicare due numeri: la varietà di proposte esistenti era frutto di un processo per tentativi in cui ognuno cercava di individuare quello che più gli si confaceva.

Un interessante schema a reticolo era ad esempio usato nei paesi arabi. In Italia era conosciuto come moltiplicazione per graticola o per gelosia, dal nome con cui veniva indicata la persiana. La gelosia, serramento per finestre a telaio fisso, consentiva ai mariti di sottrarre alla vista indiscreta di estranei le loro mogli senza impedire loro di guardare fuori.

"El sexto modo di multiplicare e chiamato gelosia ouer per graticola. E chiamase per questi nomi, perche la dispositione sua quando si pone in opera torna a moda di graticola ouer di gelosia. Gelosia intendiamo quelle graticelle che si costumono mettere ale finestre de le case doue habitano donne acio non si possino facilmente vedere o altri religiosi di che molto abonda la excelsa cita de Vinegia. E non e maraveglia chel vulgo habi trovato questi vocabuli a tali operationi, peroche ancora li astronomi hano asumpto el nome de molte stelle e siti loro, da animali e forme terrestri materiali.” [Pacioli, 1494]

Il metodo era caratterizzato dalla facilità della sua applicazione, come mostra il seguente esempio che illustra la moltiplicazione di 625 per 246.

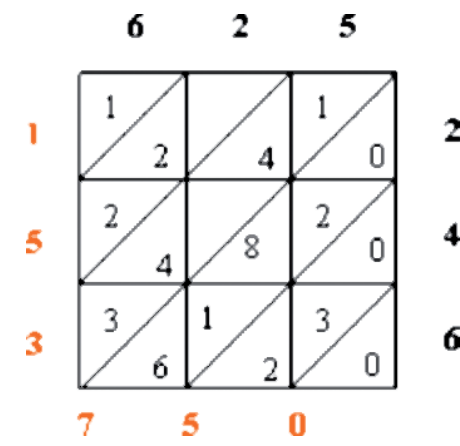


- Dida dida dida dida dida dida dida dida dida dida dida dida dida

I due numeri da moltiplicare vengono scritti ai lati di una tabella, con tante righe e tante colonne quante sono le cifre dei due fattori. In ogni cella della tabella, viene poi tracciata la diagonale principale che suddivide la cella stessa in due triangoli destinati a contenere i risultati parziali della moltiplicazione.

In ciascuna cella si scrive il prodotto parziale, cioè il risultato della moltiplicazione delle cifre dei fattori che identificano la riga e la colonna che si incrociano in corrispondenza della cel-

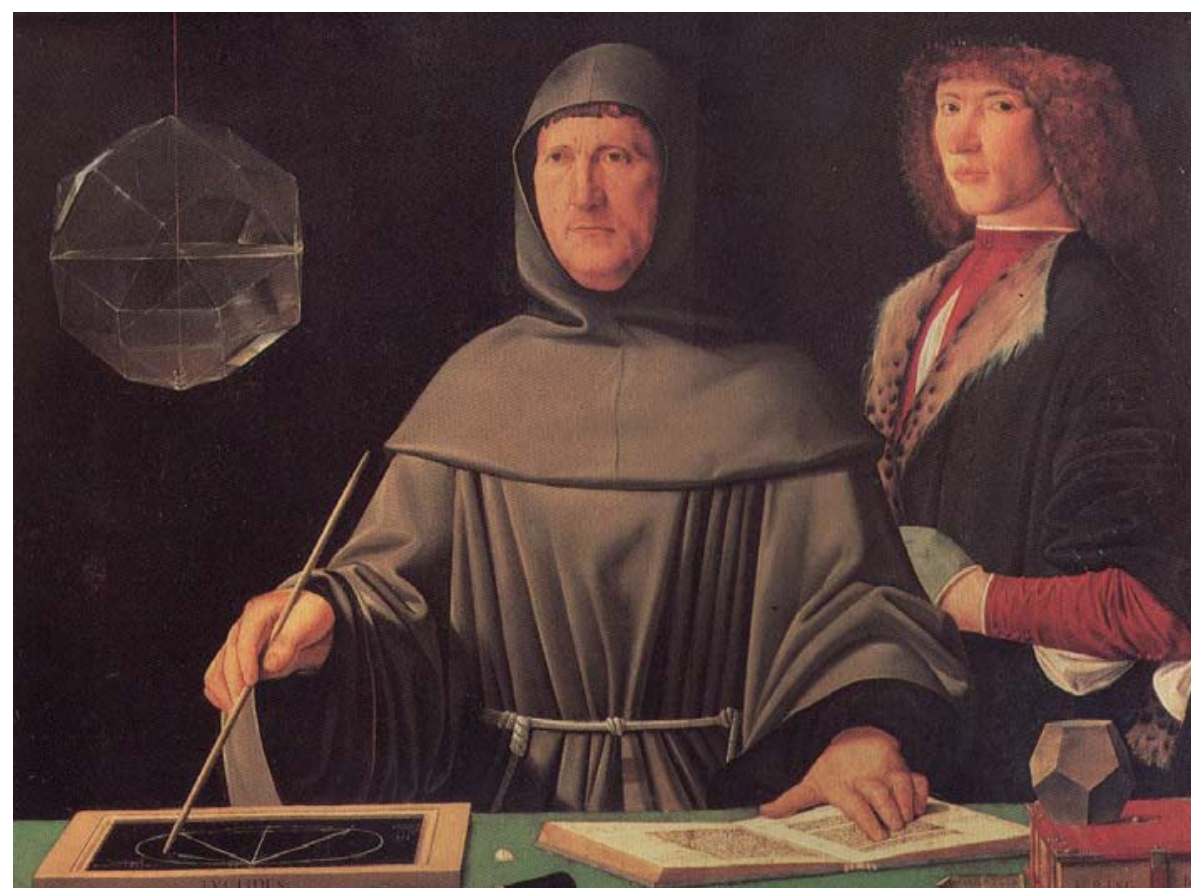
la considerata. Si pongono le decine nel triangolo superiore e le unità nel triangolo inferiore. Si sommano poi i numeri scritti nelle strisce in diagonale, considerando eventuali riporti, a partire dall'ultima striscia in basso e a destra e scrivendo in corrispondenza della striscia il risultato ottenuto (quello che nella figura è evidenziato in rosso). Il risultato finale, 153.750, è rappresentato dalla lettura dei numeri illustrati sul fianco sinistro della tabella - dall'alto in basso - e sul lato inferiore della stessa, da sinistra a destra. Tale metodo - come nota il Pacioli - "in parte se fa con lo precedente ditto quadrilatero, ma in quello se teniua le decine $e$ in questo se mette sempre tutto e poi se recogli, pure in eschincio", cioè effettuando le somme in diagonale.

Manuali in cui si presentano in maniera dettagliata semplici regole per eseguire le operazioni aritmetiche e la loro applicazione a problemi di natura pratica (regola del tre, regola di falsa posizione, ecc.) continuarono ad essere stampati anche nei due secoli successivi, XVII e XVIII. Ne sono un

esempio la Novissima prattica d'aritmetica mercantile di Domenico Griminelli, sacerdote da Correggio, e il Trattato aritmetico di Giuseppe Maria Figatelli. Pur essendo consapevoli che "molti Autori habbino scritto eccellentemente di questa materia", tali manuali venivano scritti "hauendo sempre riguardo alla breuità e faci- lità". In fin dei conti, come osservato dal Griminelli, se "in vna insalata ci venisse aggiunto il basilico o qualche altra erba buona non guastarebbe la detta insalata, ma gli accrescerebbe sapore, e fragranza d'odore, così questa operetta non pregiudicando a nessun'altra potrebbe essere di giouamento alli principianti”.

\section{BIBLIOGRAFIA}

Bagni G.T., "Il primo manuale di matematica stampato al mondo: Larte de labbacho (Treviso, 1478)", Cassamarca, 1995.

Bagni G.T., "Larte de labbacho (l’Aritmetica di Treviso, 1478) e la matematica medievale", I Seminari dell'Umanesimo Latino 2001-2002, Fondazione Cassamarca, Antilia, Treviso.

Boyer C.B., Storia della matematica, Mondadori, Milano, 1997.

Figatelli G.M., Trattato aritmetico, Venezia, 1774.

Funari S., "Quando si moltiplicava per gelosia”, in Dal Commercio all'Economia, il luogo, l'architettura e le collezioni della biblioteca di San Giobbe, Biblioteca di Economia, Università Ca' Foscari, Venezia, 2007.

Giusti E., Maccagni C., Luca Pacioli e la matematica del Rinascimento, Giunti, Firenze, 1994.

Griminelli D., N ovissima prattica d'aritmetica mercantile, Roma, 1670.

Pacioli L., Summa de arithmetica, geometria, proportioni et proportionalita, Paganino de' Paganini, Venezia, 1494 